\title{
Mesas de concertación y seguimiento de los acuerdos de paz en Guatemala: lecciones aprendidas para la paz territorial en Colombia*
}

Forums for consultation and follow-up of the peace agreements in

Guatemala: lessons learnt for territorial peace in Colombia

Mesas redondas e seguimento dos acordos de paz na Guatemala: lições aprendidas para a paz territorial na Colômbia

\footnotetext{
${ }^{*}$ Ph.D. en Estudios Internacionales de la Universidad de Cambridge (área: estudios de paz y conflicto). Profesora investigadora del Departamento de Estudios Internacionales y Comunicación de FLACSO Ecuador. Coordinadora de la línea de investigación en paz y conflicto, y responsable de la maestría de investigación en Relaciones Internacionales. Correo electrónico: camouly@flacso.edu.ec|ORCID: oooo-0oo2-0499-8213.

** Este artículo de reflexión es resultado del proyecto de investigación titulado Iniciativas locales de construcción de paz en Nicaragua y Guatemala, financiado por la Facultad Latinoamericana de Ciencias Sociales (FLACSO) Ecuador, que inició en 2011 y dio seguimiento a una investigación doctoral realizada entre 2000 y 2004. Incluye algunas reflexiones presentadas en la ponencia 'Peace constituency' or 'peace infrastructure': yeast or cobweb? en la conferencia de la Asociación de Estudios Internacionales en San Francisco, Estados Unidos en 2013. Artículo de investigación recibido el 29/01/2016 y aceptado el 17/07/2016.
} 


\section{Cómo citar}

Mouly, C. A. (2016). Mesas de concertación y seguimiento de los acuerdos de paz en Guatemala: lecciones aprendidas para la paz territorial en Colombia. Revista CS, no. 19, pp. 115-140. Cali, Colombia: Facultad de Derecho y Ciencias Sociales, Universidad Icesi.

DOI: http://dx.doi.org/10.18046/recs.i19.2141 


\section{Resumen}

Abstract

Resumo

Este artículo reflexiona sobre la experiencia de las mesas de concertación y seguimiento de los acuerdos de paz en Guatemala y saca lecciones para la paz territorial en Colombia. Basado en datos provenientes de observación, entrevistas y documentos, analiza cómo las mesas de concertación evolucionaron hasta conformar una infraestructura para la paz y el papel que desempeñaron en la descentralización de la implementación de la paz. A partir de esta experiencia, se recalca la importancia del nivel departamental como bisagra entre las bases y los tomadores de decisión a nivel central, y de la participación de grupos tradicionalmente marginados para la paz territorial. Además, sugiere tomar en consideración las iniciativas locales de construcción de paz exitosas que surgieron durante la guerra. También se discute el rol de espacios multisectoriales en la transformación pacífica de los conflictos locales y la necesidad de mantener un equilibrio entre institucionalización y flexibilidad.

\section{PALABRAS CLAVE:}

Sociedad civi | implementación de la paz | infraestructura para la paz | Guatemala | paz territorial | Colombia

This article considers the experience of the forums for consultation and the follow-up of the peace agreements in Guatemala and draws lessons for territorial peace in Colombia. Based on data from observation, interviews, and documents, it analyses how the consultation forums evolved into an infrastructure for peace, and the role they played in the decentralization of peace implementation. From this experience, It emphasizes the importance of the departmental level as a bridge between the grassroots and decision makers at the central level, and of the participation of traditionally marginalized groups for territorial peace. Additionally, the successful local peacebuilding initiatives that emerged during the war must be considered. It also discusses the role of multisectoral spaces in the peaceful transformation of local conflicts, and the need to maintain a balance between institutionalization and flexibility.

\section{KEYWORDS:}

Civil society | peace implementation | infrastructure for peac | Guatemala | territorial peace | Colombia 
Este artigo faz uma reflexão sobre a experiência das mesas redondas e seguimento dos acordos de paz na Guatemala e obtém lições para a paz territorial na Colômbia. Baseado em dados provenientes de observação, entrevistas e documentos, analisa como as mesas redondas evoluíram até conformar uma infraestrutura para a paz e o papel que desempenharam na descentralização da implementação da paz. A partir desta experiência, se reforça a importância do nível departamental como elo entre as bases e os tomadores de decisão a nível central, e da participação de grupos tradicionalmente marginados para a paz territorial. Além do mais, se deve levar em consideração as iniciativas locais de construção de paz bem sucedidas que surgiram durante a guerra. Também se discute o papel de espaços multissetoriais na transformação pacífica dos conflitos locais e da necessidade de manter um equilíbrio entre institucionalização e flexibilidade.

PALAVRAS CHAVES:

Sociedade civi | implementação da paz infraestrutura para a paz Guatemala | paz territorial | Colômbia 


\section{Introducción}

Guatemala firmó la paz en 1996, poniendo fin a más de tres décadas de conflicto armado interno. La guerra opuso el Estado a varios grupos insurgentes que se unieron bajo la Unidad Revolucionaria Nacional Guatemalteca (URNG) en 1982. Entre las raíces del conflicto estaban la exclusión política, la discriminación étnica y las profundas desigualdades socioeconómicas. Frente a la exclusión de grandes sectores de la población, los acuerdos de paz plantearon la generación de espacios que permitieran ampliar la participación social para poner fin a la "polarización económica, social y política" (Acuerdo sobre aspectos socioeconómicos y situación agraria, 1996: artículo 2). Los acuerdos de paz también previeron la creación de varias instituciones para apoyar la implementación de los mismos. Este conjunto de instituciones adquirió el nombre de "institucionalidad de la paz". Sin embargo, los negociadores no pensaron en incluir en los acuerdos la creación de un mecanismo institucional para descentralizar la implementación de la paz.

La necesidad de descentralizar la implementación de la paz para tener en cuenta la diversidad territorial se hizo sentir poco después de la firma de la paz con la aparición de distintos espacios para reflexionar sobre los acuerdos de paz y generar propuestas para su implementación. Al darse cuenta de esta necesidad, la Comisión de Acompañamiento de los Acuerdos de Paz, máximo órgano de la institucionalidad de la paz, conformada por representantes de las partes en conflicto, del órgano legislativo, de la sociedad civil (con voto) y de Naciones Unidas (sin voto), decidió institucionalizar y apoyar estos espacios que se llamaron "Mesas de concertación y seguimiento de los acuerdos de paz".

La experiencia de las mesas de concertación de Guatemala ofrece varias lecciones aprendidas para Colombia, en vista de la probable próxima firma de acuerdos de paz entre el Gobierno y las guerrillas de las Fuerzas Armadas Revolucionarias Colombianas (FARC). En el acuerdo marco para las negociaciones entre ambas partes, dentro del punto de la agenda sobre implementación, verificación y refrendación, se mencionó la creación de un "[s]istema de implementación, dándole especial importancia a las regiones" (Acuerdo general, 2012: artículo V.6.1). En este contexto se empezó a hablar de paz territorial como aspecto esencial para lograr una paz incluyente y duradera. Este artículo analiza la experiencia de las mesas de concertación y seguimiento de los acuerdos de paz (de ahora en adelante "mesas de concertación") en Guatemala y reflexiona sobre las implicaciones para la paz territorial en Colombia. Guatemala, al igual que Colombia, sufrió un largo conflicto armado producto del cierre del espacio político, de las desigualdades socioeconómicas, del acceso inequitativo a la tierra, así como de las disparidades regionales y brechas entre áreas rurales y urbanas, con una débil presencia estatal en áreas rurales periféricas. En el caso de Guatemala, además, la discriminación étnica en contra de los pueblos indígenas fue un factor crucial. 
La reflexión está articulada en torno a tres tiempos. Un primer tiempo, se enfoca en la estructura descentralizada de las mesas de concertación y en la posibilidad de construir una infraestructura para la paz que permita abarcar la diversidad territorial en la implementación de la paz. El segundo tiempo examina la importancia de la participación de grupos tradicionalmente marginados en la construcción de la paz para abordar la violencia estructural y cultural que se encuentra en la raíz del conflicto armado, atender las necesidades particulares de los distintos grupos sociales y reconocer el papel que han tenido sus integrantes en la construcción de la paz. También discute el rol de este tipo de espacios multisectoriales en la transformación pacífica de los conflictos locales y la posible participación de ex guerrilleros en estos espacios como una forma de participación en la vida pública. En un tercer tiempo, sopesa las ventajas y desventajas de la institucionalización o informalidad de este tipo de espacios y sus implicaciones para la paz territorial en Colombia.

El artículo se basa en datos obtenidos durante varias estadías de trabajo de campo en distintos lugares de Guatemala en los años 2002, 2003 y 2012, durante las cuales realicé más de 70 entrevistas semi-estructuradas en 21 de los 22 departamentos de Guatemala y observé reuniones y actividades de las mesas de concertación en los niveles nacional, departamental y municipal durante más de cuatro meses ${ }^{1}$. En menor medida, usa insumos recogidos durante estadías de trabajo de campo en Colombia. Además, utiliza datos provenientes de documentos de las propias mesas de concertación, artículos de periódicos, una amplia revisión de la literatura académica y otros documentos relevantes.

\section{Las mesas de concertación: una infraestructura para la paz descentralizada}

Las mesas de concertación aparecieron después de la firma de la paz entre el Gobierno guatemalteco y la URNG a finales de 1996, como espacios ad hoc de reflexión y propuesta sobre los acuerdos de paz. Surgieron principalmente a nivel departamental, aunque una de las primeras mesas y de las más exitosas, la mesa de concertación de occidente, abarcó inicialmente tres departamentos. Al institucionalizarse, las mesas de concertación adoptaron un mismo nombre en 2001 y una definición común como "espacio democrático, plural, multisectorial y respetuoso de la diversidad, con carácter reflexivo y propositivo que utiliza mecanismos de concertación para incidir en el cumplimiento

\footnotetext{
1. Entrevisté a miembros de las mesas de concertación de los 20 departamentos donde existían(todos los departamentos a excepción del departamento de Guatemala, correspondiente a la capital, y de Baja Verapaz), así como a personas externas que conocían esta experiencia, incluyendo a funcionarios de la Misión de Naciones Unidas en Guatemala (MINUGUA), de la Comisión de Acompañamiento de los Acuerdos de Paz, de la Secretaría de la Paz, donantes, académicos y miembros de distintas organizaciones de la sociedad civil.
} 
de los Acuerdos de Paz" (Mesas de Sacatepéquez, Quetzaltenango, Suchitepéquez, \& El Progreso, 2001). ${ }^{2}$

En el año 2003 la asamblea de mesas de concertación aprobó unos estatutos y creó una coordinadora nacional de mesas de concertación (CONAMEC) que representaba las mesas de concertación a nivel nacional. En ese momento las mesas de concertación departamentales también empezaron a promover la creación o fortalecimiento de mesas a nivel municipal (véase organigrama en figura 1). De esa forma llegaron a constituir una infraestructura para la paz de alcance nacional. Se entiende como infraestructura para la paz una "red dinámica de estructuras o mecanismos de construcción de paz interdependientes que trascienden las divisiones y los niveles de una sociedad" (Mouly, 2013: 49-50). En algún momento había mesas departamentales de concertación en cada uno de los 22 departamentos del país a excepción de la ciudad de Guatemala y del departamento de Baja Verapaz, aunque algunas de ellas eran incipientes. La CONAMEC, conformada por representantes de siete mesas departamentales de concertación, empezó a asumir la vocería de las mesas como contraparte de instituciones nacionales e internacionales, al representar las mesas en las reuniones del Grupo Consultivo para Guatemala, por ejemplo.

La expansión de las mesas a nivel municipal no llegó a tener mayor alcance porque en 2002 el Congreso aprobó el decreto que reactivó los consejos locales de desarrollo en cumplimiento con los acuerdos de paz (Congreso de la República de Guatemala, 2002), y con su aplicación distintos actores percibieron una duplicidad de funciones entre las mesas municipales de concertación y los consejos municipales de desarrollo, ya que estos últimos también fueron concebidos como espacios multisectoriales de concertación. A nivel departamental el problema fue menor porque varias mesas no incluían a representantes del estado - o dejaron de incluirlos-y se constituyeron como espacios de fortalecimiento de la sociedad civil para incidir en los consejos departamentales de desarrollo, los cuales servían de espacio de interlocución entre Estado y sociedad civil para identificar y priorizar proyectos de desarrollo a nivel local. En algunos departamentos la mesa departamental de concertación inclusive asumió la representación de la sociedad civil en el consejo departamental de desarrollo (entrevistas con miembros o ex miembros de mesas de concertación y actores externos, 2003 y 2012).

La espontaneidad con la que surgieron algunos de los primeros espacios y la durabilidad de varias mesas de concertación revelan la necesidad sentida por la sociedad civil en varios departamentos de desempeñar un papel en la implementación de la paz 
\begin{tabular}{l|l} 
FIGURA 1 & La institucionalidad de la paz en Guatemala
\end{tabular}

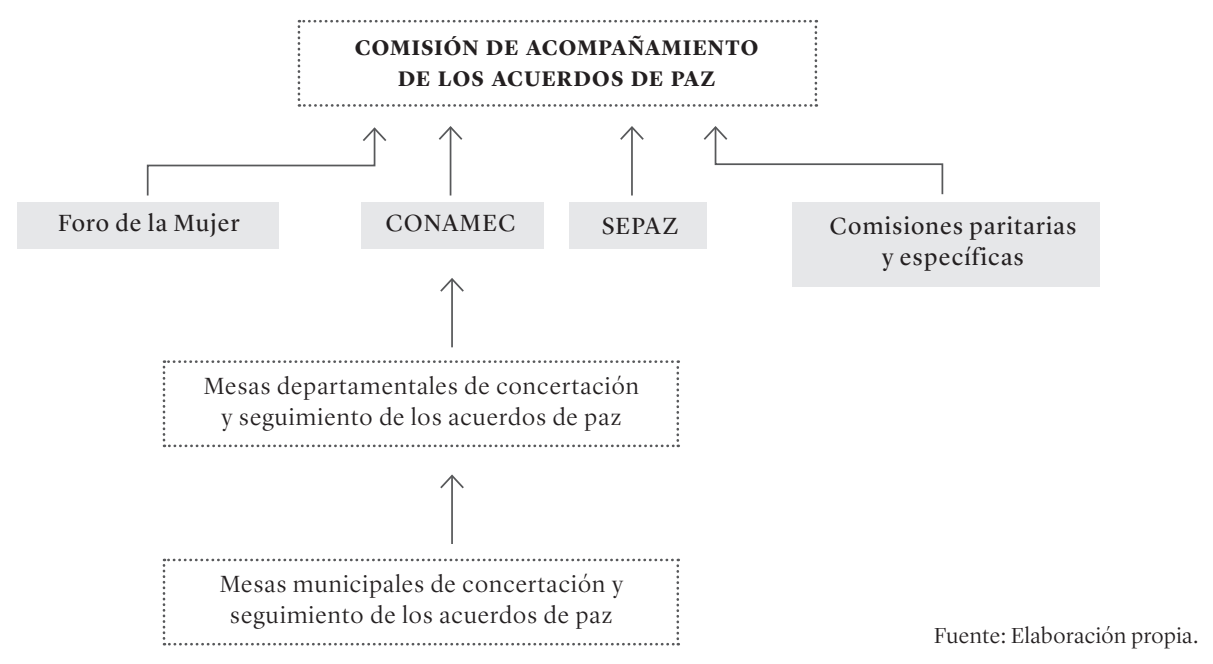

(entrevistas con miembros de mesas de concertación, ex miembros de la Comisión de Acompañamiento de los Acuerdos de Paz, ex secretario técnico de la Secretaría de la Paz y ex funcionaria de la Misión de Naciones Unidas en Guatemala, 2002). Las mesas aportaron insumos importantes para la implementación de la paz desde la perspectiva de su localidad. Hicieron llegar propuestas a la Comisión de Acompañamiento y Seguimiento de los Acuerdos de Paz y participaron en espacios de discusión sobre la implementación de los acuerdos de paz, como en los encuentros nacionales de la institucionalidad de la paz y en la Comisión de Seguimiento del Pacto Fiscal, donde se discutió la implementación de las reformas tributarias contempladas en el Acuerdo sobre aspectos socioeconómicos y situación agraria.

Las mesas desempeñaron un papel clave en la discusión de muchos aspectos de los acuerdos de paz, como la participación social o las reformas para garantizar los derechos de los pueblos indígenas, que solamente estaban esbozados de forma general y necesitaban ser precisados para implementarse. En efecto, a pesar de que las negociaciones de paz en Guatemala duraron más de seis años con amplias consultas a la sociedad civil, no llegaron a abordar todos los temas a fondo y la implementación de la paz requirió una discusión más profunda de muchos temas³. Por lo tanto, las mesas

3. Por eso, los acuerdos de paz contemplaron la creación de espacios temporales, como el Foro de la Mujer o las comisiones paritarias y específicas, para discutir la implementación de distintas provisiones de los acuerdos de paz, como la participación de la mujer, el acceso a la educación bilingüe para los pueblos indígenas o las reformas electorales. 
sirvieron de espacio para que dialogaran los diferentes sectores representados en ellas y, conjuntamente, formularan propuestas para la implementación de los acuerdos desde la realidad departamental.

Además de hacer llegar sus propuestas a la Comisión de Acompañamiento de los Acuerdos de Paz, varias mesas organizaron encuentros con diputados del departamento para remitirles sus puntos de vista sobre iniciativas de ley relevantes y entregarles sugerencias. Durante los periodos electorales muchas organizaron foros públicos con la participación de candidatos a diputado a quienes se preguntaba sobre sus compromisos con la implementación de varios aspectos pendientes de los acuerdos de paz importantes para el departamento; algunas inclusive se reunieron con candidatos a presidente de la república (entrevistas con miembros y ex miembros de mesas de concertación, 20022003 y 2012). En Quetzaltenango, por ejemplo, la mesa de concertación organizó tres encuentros con partidos políticos antes de las elecciones generales de 2003. Durante el tercero (ver foto 1) la mesa hizo firmar a los representantes de los partidos un documento en el que se comprometían, en nombre de su partido, a respetar la ley de consejos de desarrollo, abrir los gobiernos municipales a la participación de sectores marginados,

\begin{tabular}{|l|l|}
\hline FOTо 1 & Encuentro con candidatos \\
\hline
\end{tabular}

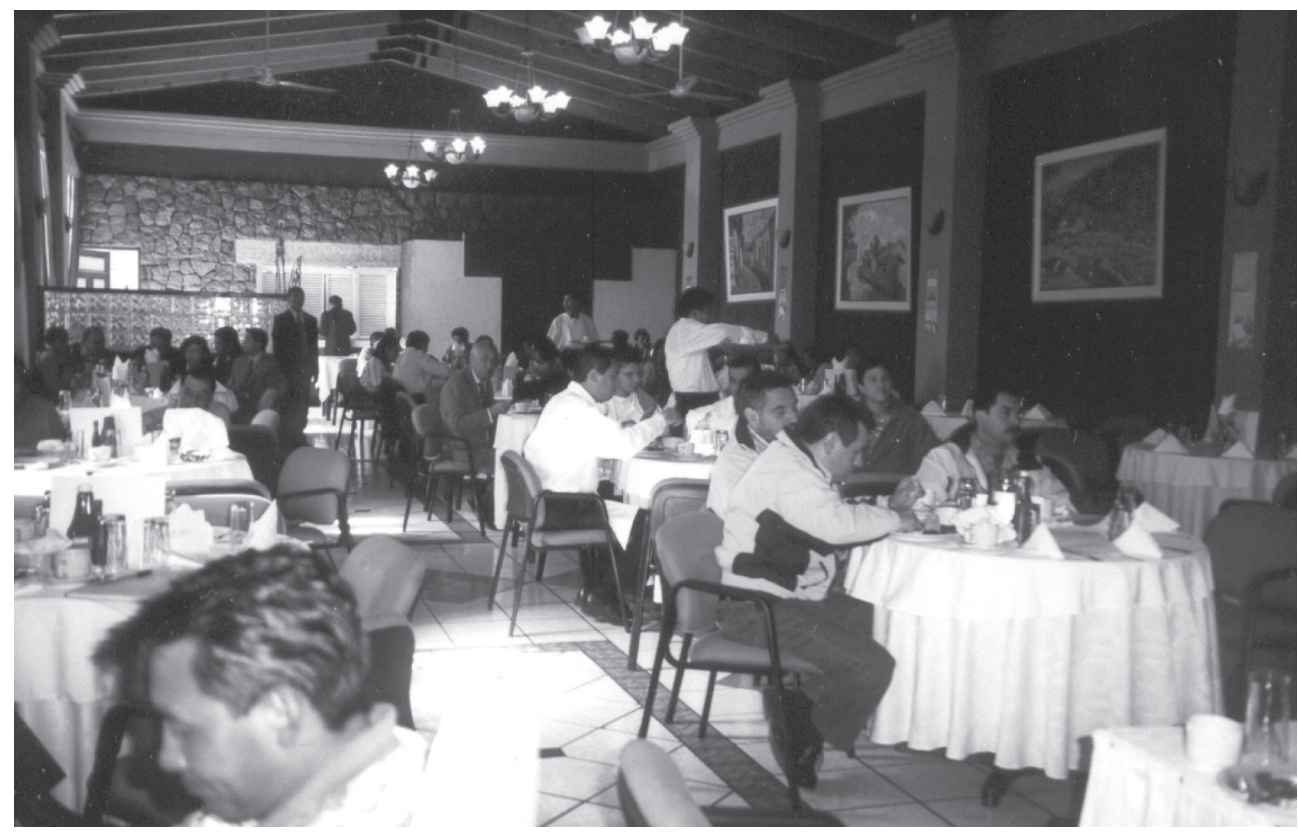

Fuente: Encuentro con candidatos a alcalde y diputado de cinco partidos y movimientos políticos, medios de comunicación y organizaciones de la sociedad civil, organizado por la mesa departamental de Quetzaltenango el 11 de septiembre de 2003. Foto tomada por la autora. 
elaborar una ley que convirtiera los acuerdos de paz en acuerdos de estado y respetar la equidad de género, edad y representación étnica (ver notas 79 y 80 de trabajo de campo).

En un país diverso y desigual, con departamentos con un alto porcentaje de población indígena y otros casi sin población indígena, con departamentos altamente urbanizados y otros principalmente rurales, las mesas permitieron dar cuenta de las diferencias territoriales en la implementación de la paz. Así, la mesa departamental de concertación de Sololá, cuya población era indígena en más del 90\% en la posguerra (PNUD, 1998: 220), dio prioridad a la implementación del Acuerdo sobre identidad y derechos de los pueblos indígenas. Mientras tanto, la mesa de concertación del Quiché, uno de los departamentos más afectados por el conflicto armado, donde se cometieron actos de genocidio de acuerdo al informe de la comisión de la verdad (Comisión de Esclarecimiento Histórico, 1999), puso énfasis en los derechos humanos y en el tema de las víctimas. De forma parecida, los desplazados internos desempeñaron un papel destacado en la mesa de concertación del Petén, departamento con un alto número de desplazados (entrevistas con representantes de las mesas de Sololá, Quiché y el Petén, 2002-2003).

Además, las mesas de concertación generaron una mayor apropiación de los acuerdos de paz por parte de la población. Casi todas las mesas organizaron eventos de difusión de los acuerdos de paz a nivel departamental y en los distintos municipios del departamento. En estos eventos no solamente explicaron el contenido de los acuerdos de paz, sino también contestaron los interrogantes del público y recogieron propuestas. Según varios autores (ej. Donais, 2009), la apropiación del proceso de construcción de paz por parte de la población es clave para garantizar una paz duradera. Por eso, uno de los mayores aportes de las mesas de concertación fue permitir una amplia participación ciudadana en el debate acerca de los acuerdos de paz por medio de foros, asambleas y otras actividades.

Los aportes de las mesas de concertación en este sentido fueron notables en el caso de las mesas que funcionaban de manera más democrática. Éstas organizaban asambleas regulares con participación amplia de distintos grupos sociales que permitían generar propuestas consensuadas. La junta directiva luego se encargaba de canalizar las propuestas a las instancias pertinentes o eran compartidas directamente con funcionarios estatales invitados por las mesas para abordar temas específicos, como la descentralización (véase foto 2, por ejemplo). Sin embargo, en ausencia de reglas comunes, no todas las mesas funcionaron de manera democrática y algunas solo sirvieron para difundir las propuestas de un grupo reducido de personas con poca representatividad.

De esta experiencia podemos sacar varias lecciones sobre posibles mecanismos para descentralizar la implementación de los acuerdos de paz en el caso de la paz territorial en Colombia y sus posibles desafíos. En primer lugar, el desarrollo de una infraestructura para la paz con la participación de actores clave de los 32 departamentos de Colombia y 


\begin{tabular}{|l|l|}
\hline FOTO 2 & Asamblea de la mesa departamental de concertación de Huehuetenango \\
\hline
\end{tabular}

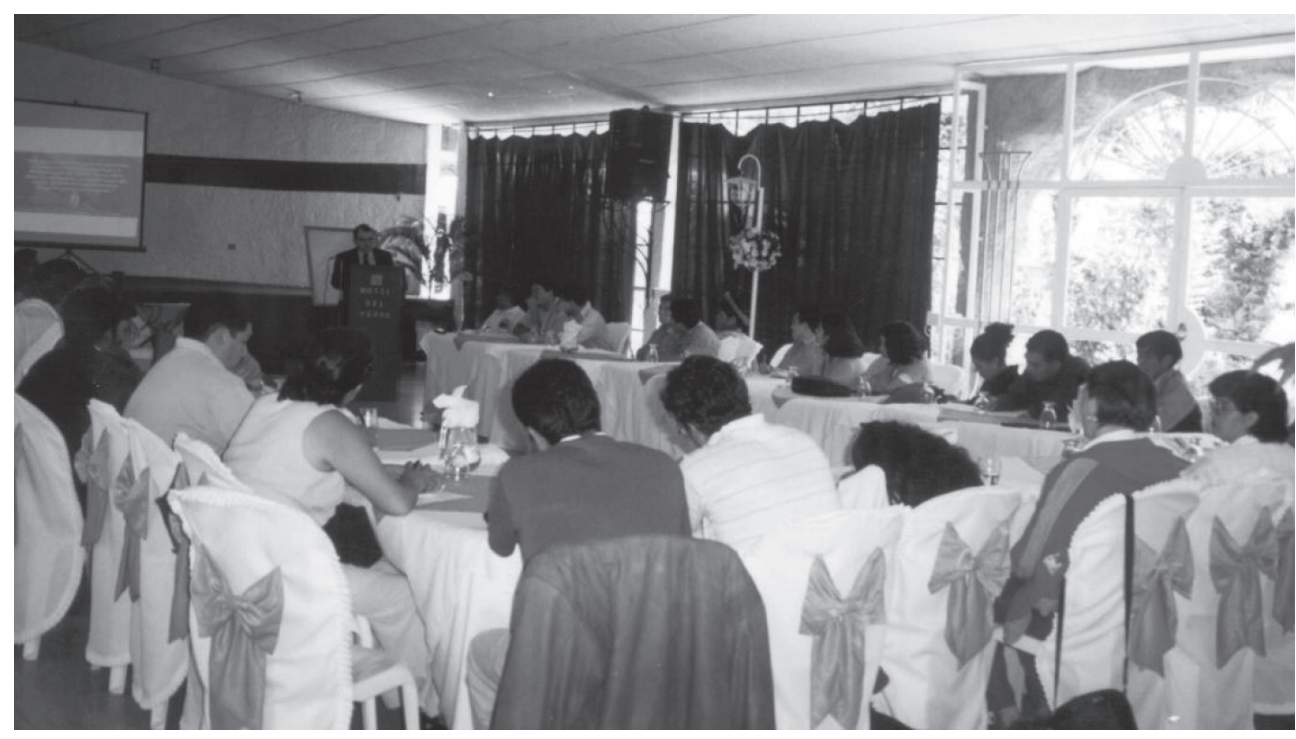

Fuente: Asamblea de la mesa departamental de concertación de Huehuetenango con la participación de representantes de la Comisión Presidencial sobre Descentralización y de la Comisión Presidencial sobre Derechos Humanos el 25 de abril de 2002. Foto tomada por la autora.

de la capital Bogotá es clave para implementar los acuerdos de paz, tomando en cuenta las diferencias territoriales en términos de afectación por el conflicto armado, diversidad poblacional, desarrollo socioeconómico, población rural y urbana, etc. Es imprescindible considerar las particularidades departamentales para construir una paz incluyente donde cada uno pueda sentirse representado en un país tan diverso como Colombia, donde según datos del censo de 2005 en el departamento de Vaupés la población indígena representa más del $66 \%$ de la población, mientras varios departamentos (ej. Valle del Cauca, Santander) tienen menos del 1\% de población indígena; asimismo, el departamento de Chocó cuenta con más de $82 \%$ de población afrocolombiana, mientras en varios departamentos (ej. Norte de Santander, Tolima) la población afrocolombiana representa menos del 2\% (DANE, s/f).

En segundo lugar, al igual que en Guatemala, el nivel departamental puede desempeñar un papel esencial en Colombia como bisagra entre las bases y el nivel nacional, donde se adoptan las leyes y se definen las políticas públicas. De esta forma, se puede fomentar lo que John Paul Lederach ha llamado la integración vertical (Lederach, 1997, 1999, 2008; véase también Journal of Peacebuilding $\mho$ Development, 10(1)). Tal integración es imperativa para que la población a nivel de base se involucre en el pro- 
ceso de construcción de paz, teniendo acceso a información sobre acontecimientos a nivel nacional, y pueda alimentar las decisiones tomadas a nivel central a partir de sus experiencias concretas a nivel local. Solamente así se puede lograr una verdadera apropiación del proceso de construcción de paz por parte de la población y, por consiguiente, una paz duradera.

En tercer lugar, es importante tomar en cuenta los mecanismos existentes para evitar duplicidades. En el caso de Guatemala el esfuerzo por crear mesas municipales de concertación quedó trunco con la creación de los consejos municipales de desarrollo, en cumplimiento con el Acuerdo sobre aspectos socioeconómicos y situación agraria (1996: artículo 10 inciso f), que hizo que muchas mesas municipales perdieran relevancia. A nivel departamental, ocurrió lo mismo, aunque en una menor medida, ya que, aun después de que la institucionalidad de la paz oficialmente terminó su labor en el año 2004, varias mesas siguieron funcionando y complementaron el trabajo de los consejos departamentales de desarrollo.

No obstante, tal como Timothy Donais (2009) hace notar, los mecanismos existentes pueden reproducir los mismos desequilibrios y falencias que los acuerdos de paz intentan corregir. Según el Alto Comisionado para la Paz en Colombia, la implementación de la paz no partirá de ceros, pero:

(...)es inevitable que [el país] tenga o haya tenido serias fallas en su institucionalidad, tanto en su capacidad de producir bienes públicos y satisfacer derechos en todo el territorio, como de asegurar las condiciones para tramitar las demandas políticas de la sociedad [...] Los obstáculos sin duda son enormes: la corrupción, el clientelismo, las redes de intereses creados y del crimen organizado amenazan cualquier intento de reconstrucción” (Jaramillo, 2014).

Entonces no hay garantías de que los mecanismos existentes en Colombia tengan la capacidad o voluntad de contribuir al proceso de construcción de paz. Si tienen esta capacidad o voluntad, deberían ser utilizados y fortalecidos en lugar de crear nuevas instancias desde cero. Si no la tienen, se requeriría, al menos temporalmente, establecer nuevos mecanismos que, por ende, deberían contribuir a la construcción de capacidades en el Estado y la sociedad (Chopra, 2009; Donais, 2009). Este diagnóstico se debería hacer con base en un sólido conocimiento de las realidades de cada departamento y en consulta con actores locales que han demostrado su compromiso por la paz.

En cuarto lugar, sería útil pensar cómo articular los distintos mecanismos institucionales a nivel departamental y garantizar una adecuada representación de los departamentos a nivel nacional. Tal articulación puede ser esencial en Colombia, país con mayor número de departamentos que Guatemala, aunque, como Adane Ghebremeskel y Richard Smith señalan, esta articulación no debe ser impuesta y ninguna entidad local debería ser obligada a coordinar esfuerzos con sus pares en otras locali- 
dades (Ghebremskel \& Smith, 2013). No obstante, si estas entidades locales deciden articularse para conformar una infraestructura de alcance nacional y así tener mayor incidencia, se debe tener cuidado de que no pase lo que ocurrió en Guatemala con la CONAMEC, cuya creación, a pesar de sus buenos propósitos, tuvo muchos desaciertos. La mayor debilidad de la CONAMEC fue su falta de representatividad; a pesar de ser conformada por representantes de un tercio de las mesas departamentales de concertación, fue constituida por representantes de muchas mesas incipientes y/o no representativas. Esto hizo que perdiera legitimidad a los ojos de la mayoría de las mesas más representativas y activas, que expresaron severas críticas en las asambleas nacionales. Por lo tanto, en caso de establecer alguna instancia de coordinación, sería esencial fijar criterios de representatividad y rendición de cuentas. En Guatemala varias mesas trataron de empujar la elaboración de estatutos para la CONAMEC, para evitar abusos, pero sus esfuerzos fueron vanos debido a la desigual correlación de fuerzas en la asamblea de mesas departamentales de concertación. En efecto, la Secretaría de la Paz (SEPAZ), institución gubernamental que fungió como secretaría ejecutiva de la Comisión de Acompañamiento de los Acuerdos de Paz, estaba encargada de convocar las mesas departamentales de concertación a las asambleas, pero convocaba a varias mesas incipientes o, peor aún, inexistentes (mesas que habían desaparecido por ejemplo) y a veces no convocaba a las mesas más consolidadas (Entrevistas con miembros o ex miembros de mesas de concertación y actores externos, 2002-2003 y 2012).

Sobre este último punto, la legitimidad de espacios como las mesas de concertación o de una futura infraestructura para la paz en Colombia no necesariamente pasa por la participación de un alto número de personas. Según John Paul Lederach (2008), la "levadura crítica" es más importante que la masa crítica. Por lo tanto, la participación de líderes con relaciones sociales que rebasen las divisiones del conflicto y puedan tender puentes entre diversos grupos, fomentando así una integración horizontal y la restauración del tejido social destruido por el conflicto armado, es quizás lo más importante para construir la paz (Lederach, 1997, 1999, 2008). Al involucrar no solamente a diferentes sectores y niveles de la sociedad sino también a líderes capaces de conectar a estos sectores y niveles, una infraestructura para la paz puede facilitar la integración horizontal y vertical de un proceso de construcción de paz (Hopp-Nishanka, 2012: 5). En las mesas departamentales de concertación algunos líderes vinculados a la iglesia católica desempeñaron un papel fundamental en este sentido. Por ejemplo, la primera coordinadora de la mesa de concertación de occidente fue una mujer vinculada a la Pastoral Social, quien contribuyó de manera determinante a la consolidación de la mesa de concertación por su intermediación entre los distintos grupos sociales representados en la mesa (entrevistas con miembros de mesas de concertación, 2002-2003). 


\section{Las mesas de concertación: espacio para la intermediación y la participación de grupos tradicionalmente marginados}

Las mesas de concertación fueron concebidas como espacios multisectoriales que reflejasen la diversidad poblacional en cada localidad. Todas incluían a representantes de diversos sectores de la sociedad civil, entendida como espacio organizativo que va más allá de la familia y se distingue del Estado y del sector privado. En muchos casos (ej. mesa del Quiché) también tenían representación del Estado, por ejemplo a través de delegaciones departamentales de ministerios o instituciones como la Procuraduría de los Derechos Humanos (equivalente de la Defensoría del Pueblo en Colombia), pero no en todos (ej. mesa de Alta Verapaz). De manera similar, en varios casos (ej. mesa de Chimaltenango) había representación del sector privado, mientras en otros (ej. mesa de Sololá) no.

Asimismo, la junta directiva de cada mesa tuvo su propia composición, en función de las particularidades locales. En Jutiapa, por ejemplo, la junta directiva comprendía a representantes de organizaciones campesinas, organizaciones de mujeres, organizaciones indígenas, sindicatos, instituciones estatales, la academia, el gremio de periodistas y la casa de la cultura. No todos estos grupos estaban representados en las distintas mesas, pero era común la apertura de las mesas a la participación de grupos tradicionalmente marginados, como pueblos indígenas, mujeres y campesinos. También fueron un espacio importante para la participación de ex guerrilleros reintegrados a la vida civil, en representación de la URNG o de otros grupos, y en algunas ocasiones de miembros de otros partidos políticos. Estos grupos sociales sacaron provecho de la implementación de la paz para conseguir mayor representación y utilizaron las disposiciones de los acuerdos de paz que exigían una mayor participación de grupos tradicionalmente marginados y una reducción de las brechas sociales (Idler, Mouly, \& Miranda, 2015).

Las mujeres fueron uno de los sectores que más provecho sacó de las mesas de concertación. En la mayoría de mesas jugaron un rol preponderante. Muchas mujeres presidieron sus mesas, como Úrsula Roldán, quien lideró la junta directiva de la mesa de concertación de Occidente durante tres años. De manera similar, algunas mujeres encabezaron las juntas directivas de sus mesas departamentales, como en los casos de Huehuetenango, Sololá, San Marcos, Izabal y El Progreso. Por otro lado, la mesa departamental de Alta Verapaz, además de incluir a varias mujeres, estableció una mesa compuesta solamente por mujeres, la cual organizó un foro con candidatos a alcalde en la capital del departamento, Cobán, en octubre de 2003, donde presentó una agenda con enfoque de género (ver notas de trabajo de campo 97). La participación de las mujeres en las mesas contribuyó al empoderamiento de muchas mujeres y permitió luchar contra el machismo imperante. En varios departamentos (ej. El Progreso) hubo una relación entre el Foro de la Mujer, institución creada en virtud de los acuerdos de 
paz para promover la participación de las mujeres, y las mesas de concertación en la medida en que algunas mujeres fueron integrantes de ambos espacios. Las organizaciones de mujeres participaron activamente en la mayoría de mesas de concertación. Estas mesas también fomentaron la participación de las mujeres en otros ámbitos. Por ejemplo, la mesa departamental de concertación de Izabal, en alianza con la Defensoría de la Mujer, promovió la participación de las mujeres en las elecciones generales de 2003 (ver notas de trabajo de campo 96). De forma parecida, en 2001, en una entrevista en la radio local, el primer secretario-general de la mesa departamental de concertación de Sololá alentó a las mujeres en participar en la vida pública y a darse cuenta "de sus conocimientos y derecho a hablar" (Quiacaín citado en MINUGUA, 2001).

Otro grupo tradicionalmente marginado que participó activamente en las mesas de concertación fue el de los indígenas. En algunas mesas, la mayoría de los integrantes de la junta directiva eran indígenas. Fue el caso de Sololá donde Leonicia Pocop, dirigente maya kaqchikel, fue electa presidente de la mesa departamental de concertación y desempeñó un papel importante en la promoción del Acuerdo sobre Identidad y Derechos de los Pueblos Indígenas (ver foto 3). En Huehuetenango, otro departamento con un alto porcentaje de población indígena, la mesa difundió los acuerdos de paz entre la población indígena y fomentó la participación de la población indígena en la implementación de la paz. A pesar de esta contribución para remediar una de las raíces

\begin{tabular}{|l|l|}
\hline FOTо $_{3}$ & Junta directiva de la mesa departamental de concertación de Sololá \\
\hline
\end{tabular}

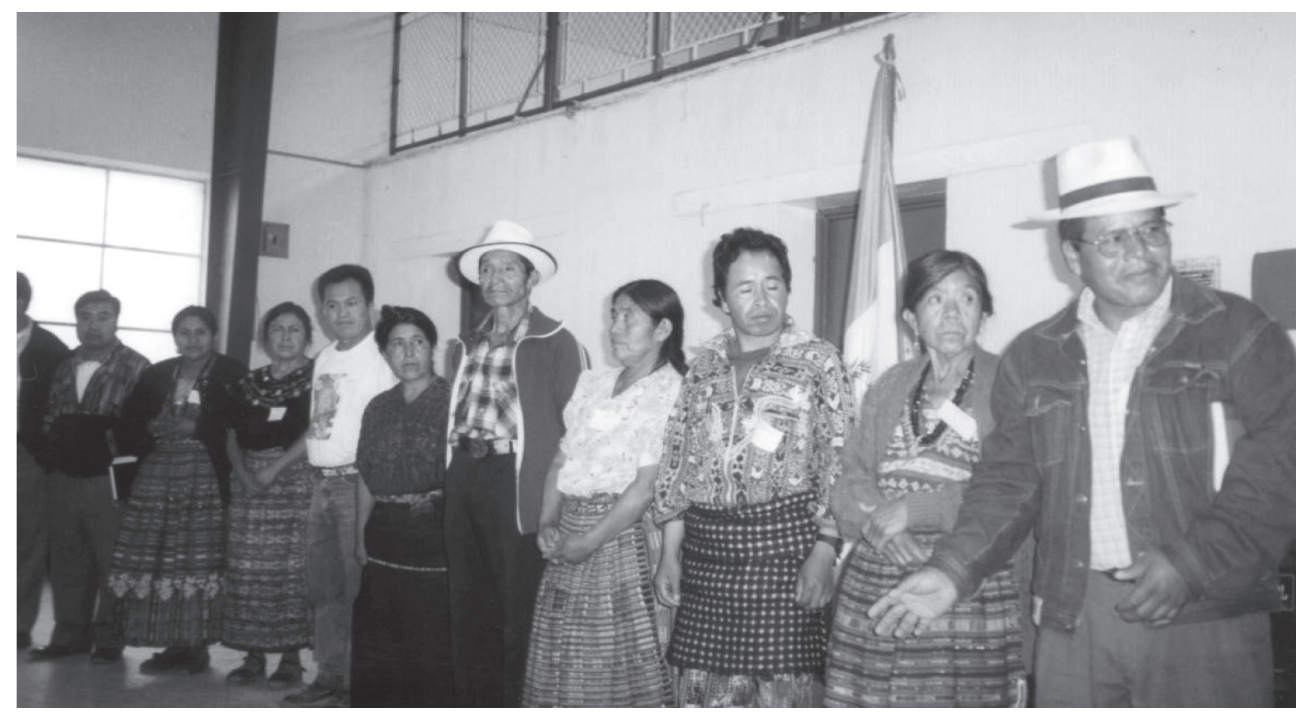

Fuente: Junta directiva de la mesa departamental de concertación de Sololá electa en mayo de 2002, integradas por 11 indígenas y un miembro no indígena. Foto tomada por la autora. 
profundas del conflicto armado, la discriminación étnica, todas las mesas funcionaban en español y realizaban sus asambleas en ese idioma. La mesa de Sololá pensó en realizar sus asambleas no solo en español sino igualmente en dos de los idiomas indígenas más hablados en su departamento, pero no lo pudo hacer por razones prácticas (Idler, Mouly, et al., 2015).

El sector campesino también tuvo representación en muchas mesas, aportando a la discusión de los temas agrarios. El presidente de la mesa departamental de Jutiapa en los años 2002-2003, por ejemplo, era el representante del sector campesino. Esta mesa sirvió de mediadora entre el Fondo de Tierras (FONTIERRAS), creado en virtud de los acuerdos de paz para fomentar el acceso a la tierra, y campesinos del departamento cuando ocuparon las oficinas de FONTIERRAS (entrevistas con representantes de la mesa de Jutiapa, 2002-2003). Esta participación fue importante, dado que la concentración de la tierra era una de las raíces del conflicto y el Acuerdo sobre aspectos socioeconómicos y situación agraria fue uno de los acuerdos más difíciles de implementar debido a la reticencia del Comité Coordinador de Asociaciones Agrícolas, Comerciales, Industriales y Financieras (CACIF), el poderoso gremio de empresarios y terratenientes.

De igual manera, en el caso de Colombia, las desigualdades socioeconómicas y la concentración de la tierra son raíces significativas del conflicto armado. Por eso uno de los seis puntos de la agenda de negociaciones entre el Gobierno y las FARC fue el tema agrario, considerado por varios analistas como central. Además, las áreas más afectadas por la violencia armada de los últimos 40 años fueron las rurales. En este contexto, la participación de la población rural en la implementación de los acuerdos de paz es clave, por lo que la paz territorial debería tomar debidamente en cuenta a este sector de la población; también es importante involucrar a las mujeres, que han sido afectadas por el conflicto armado tanto como los hombres, aunque de manera diferente. Algunas participaron activamente en el mismo y otras aportaron a la construcción de la paz. Existen múltiples organizaciones y redes de mujeres que han trabajado por la paz en Colombia (Rojas, 2009) y podrían desempeñar un papel valioso en el marco de la paz territorial.

Por otro lado, la violencia armada afectó de manera particular a los pueblos indígenas y afrodescendientes (Romero, 2015). En varias instancias estos pueblos fueron obligados a desplazarse, a pesar de su apego a la tierra. En otras se organizaron para enfrentar la violencia y evitar el desplazamiento. Entre los ejemplos más conocidos están el Colectivo de Comunidades Negras de la Asociación Campesina Integral del Atrato (COCOMACIA) y los indígenas nasa que, por medio del Consejo Regional Indígena del Cauca (CRIC), han actuado como mediadores en el conflicto armado para reducir sus efectos negativos sobre la población civil (Hernández, 2012: 105-160, 161-206; 2013). Estos pueblos tradicionalmente marginados tienen necesidades específicas que deberían ser atendidas para garantizar una paz incluyente y duradera. Por lo tanto, sería impera- 
tivo abrir espacios para su participación, sobre todo en lugares donde estas poblaciones son significativas, y reconocer y difundir experiencias exitosas como las citadas.

Un rol fundamental de las mesas de concertación fue la intermediación, al acercar a grupos opuestos (ej. URNG y Ejército) o simplemente distanciados, y fomentar el diálogo entre distintos grupos sociales. Así generaron propuestas consensuadas y contribuyeron a la construcción de una cultura de paz basada en el diálogo en lugar de la imposición. Dado que la intolerancia (forma de violencia cultural) fue una de las raíces del conflicto armado guatemalteco, esto fue en sí un aporte a la construcción de la paz. De la misma forma, este tipo de espacios de concertación a nivel local en Colombia puede ser fundamental para la paz territorial y la reconciliación (Jaramillo, 2014).

Además de ser espacio de interlocución entre diversos grupos sociales, permitiendo abordar conflictos potenciales o actuales entre estos, algunas mesas mediaron en conflictos locales. Por ejemplo, la mesa de concertación de San Marcos medió en el conflicto de tierra de la finca Australia y la mesa de concertación de occidente entre los alcaldes comunales del departamento de Totonicapán y el Gobierno luego de un conflicto violento que resultó en la declaración del estado de sitio. Mientras tanto, la mesa departamental de Huehuetenango convocó una mesa de diálogo entre la asociación de transportistas de Huehuetenango, la Procuraduría de los Derechos Humanos y víctimas luego de un accidente de bus (entrevistas con miembros de las mesas de concertación de San Marcos, occidente y Huehuetenango, 2002-2003 y 2012). Muchos conflictos suelen estallar durante los periodos post acuerdos, en particular conflictos de tierra relacionados con el retorno de las poblaciones desarraigadas. En el caso de Guatemala algunas mesas, como la mesa de negociaciones para conflictos de tierra de Alta Verapaz (o "mesa de tierra de Alta Verapaz" en corto), abordaron específicamente los conflictos de tierra y fueron exitosas en transformar estos conflictos de manera pacífica, evitando que se tornaran violentos (Aguilar, 2002).

En el caso colombiano también es probable que surjan múltiples conflictos. La transformación pacífica de los conflictos de tierra, en especial, será un desafío clave del periodo post acuerdos (Romero, 2015). Los conflictos vinculados al retorno de las poblaciones desarraigadas, a la explotación de recursos naturales o a la ausencia de títulos de propiedad pueden tornarse violentos, si no son abordados oportunamente. Por lo tanto, algunas lecciones se podrían sacar del caso guatemalteco, en general, y de la experiencia de la mesa de tierra de Alta Verapaz, en particular. Esta última convocó a amplios sectores de la sociedad y a las partes en conflicto para encontrar salidas pacíficas a múltiples conflictos y gozó de mucha legitimidad en el departamento (Aguilar, 2002). La creación de este tipo de espacios informales multisectoriales podría ser útil en departamentos con muchos conflictos de tierra donde las capacidades institucionales para abordar estos conflictos son insuficientes, carecen de legitimidad para tratar ciertos conflictos o no son lo suficientemente ágiles para evitar que estos se tornen violentos. 
No obstante, sería necesario adoptar ciertos procedimientos que fomenten la empatía entre los participantes para asegurar que estos espacios contribuyan efectivamente a la transformación pacífica de los conflictos en lugar de exacerbarlos. Como muestran Juan Ugarriza y Enzo Nussio en un estudio reciente con ex combatientes, víctimas y miembros de la comunidad en nueve municipios de Colombia, el simple contacto entre las partes antagónicas no garantiza una mejora de sus relaciones; las condiciones en que los acercamientos se dan inciden en el resultado (Ugarriza y Nussio, 2016). Esto también permite explicar por qué, si bien las mesas de concertación contribuyeron generalmente a la transformación pacífica de los conflictos, no fue siempre el caso. Así, poco después de su conformación, la mesa de concertación de occidente, por ejemplo, fue el foco de un conflicto interno entre el ejército, por un lado, y las organizaciones sociales, por otro, luego de que la mesa organizó una marcha en contra de los asesinatos del Obispo Juan Gerardi y de una fiscal de Quetzaltenango en 1998. A raíz de este conflicto, el ejército dejó de participar en la mesa durante varios meses (entrevista con ex miembro de la mesa de concertación de occidente, 2002). Posteriormente la mesa desempeñó un papel clave en la mediación del conflicto entre las autoridades estatales y las autoridades comunales e indígenas de Totonicapán, entre otros conflictos (Mouly, 2004: 273-274).

Finalmente, uno de los temas más sensibles es la participación de ex guerrilleros. No todos los insurgentes que se desmovilicen y quieran participar en la vida pública podrán hacerlo por la vía electoral, aun con la creación de circunscripciones electorales especiales. En Guatemala la falta de oportunidades de participación por medios electorales hizo que muchos ex miembros de la URNG se voltearan hacia espacios de la sociedad civil para contribuir a la vida pública, incluyendo las mesas de concertación. En la mesa de concertación de occidente la junta directiva llegó a ser integrada en algún momento por una mayoría de ex miembros de la URNG representando diversos sectores: la URNG, las ONG, la academia, etc.; lo que fue problemático en cuanto la mesa empezó a politizarse (entrevistas con miembros y ex miembros de la mesa de concertación de occidente y actores externos, 2002-2003 y 2012). No obstante, la posibilidad para ex guerrilleros de tener voz por medio de su participación en la sociedad civil puede ser clave para garantizar una paz duradera, en la cual la gente desista de las armas para hacer oír su voz. De acuerdo al Alto Comisionado para la Paz en Colombia, "si estamos pensando en transformar los territorios y crear institucionalidad sobre la base de la participación y la inclusión, estos procesos no se pueden limitar a ofrecerles oportunidades de vida a quienes han dejado las armas. Tienen que ser parte integral del proceso de reconstrucción territorial" (Jaramillo, 2014). Por consiguiente, una participación equilibrada del Estado, de los ex movimientos insurgentes, de los distintos grupos que conforman la sociedad civil y del sector privado en espacios de concertación a nivel departamental puede contribuir al éxito de la paz territorial. 


\section{¿Flexibilidad o institucionalización?}

Otro punto que merece ser discutido en relación a los mecanismos para la implementación de los acuerdos de paz es si deben ser flexibles o institucionalizados. Esta discusión cobra relevancia en el caso de las mesas de concertación, que pasaron de ser espacios informales a espacios institucionalizados con el apoyo de la Comisión de Acompañamiento de los Acuerdos de Paz y su incorporación en la institucionalidad de la paz. También es útil para pensar la mejor manera de asegurar la sostenibilidad de la paz territorial, sin perder la capacidad de adaptarse al contexto fluctuante de posguerra.

Las situaciones post acuerdos tienden a ser volátiles. Un cambio de gobierno o en el entorno internacional puede tener repercusiones significativas sobre las posibilidades de implementar los acuerdos de paz. En estas situaciones, iniciativas informales como las mesas de concertación, que no requieren muchos recursos, pueden tener más durabilidad. No obstante, también pueden ser más efímeras por la carencia de recursos económicos. Dicha carencia puede ser tanto una bendición como una maldición. En el caso de las mesas de concertación, el apoyo de varias instituciones nacionales e internacionales ayudó a fortalecer las mesas, pero, al mismo tiempo, generó una dependencia y abusos en algunos casos. Además, con frecuencia los donantes terminan incidiendo en la agenda de las iniciativas que financian (directamente o indirectamente) por el poder que ejercen (ej. Ghebremskel y Smith, 2013). Por lo tanto, se requiere cautela al momento de asignar recursos a iniciativas locales de paz para no desvirtuarlas.

Por otro lado, aunque las instituciones suelen tener de por sí mayor durabilidad, la institucionalización puede conllevar una pérdida de vinculación con las bases, haciendo que espacios que anteriormente gozaban de mucha legitimidad rápidamente pierdan relevancia, como lo muestra el ejemplo de algunas mesas que decayeron por estos motivos (Idler, Mouly, et al., 2015). Mientras tanto, mesas como la mesa de concertación de occidente perduraron por más de diez años porque lograron desempeñar un rol pertinente y subsistir pese a los cambios de líderes. Las iniciativas informales suelen tener mayor adaptabilidad: pueden cambiar de forma y fortalecer iniciativas más formales. Esto ocurrió con varias mesas que desaparecieron una vez que los consejos de desarrollo fueron lo suficientemente consolidados. Los miembros de las juntas directivas de estas mesas siguieron activos en estos consejos y/u otros espacios. De la misma forma, varios miembros de las comisiones de paz en Nicaragua se convirtieron en facilitadores judiciales, reconocidos por el ministerio de Justicia, con la aparición de esta nueva figura (Mouly, 2013).

Además, debemos reflexionar sobre las implicaciones de una estructura informal o institucionalizada en cuanto a las posibilidades de implementar la paz. Por un lado, los espacios informales, como las mesas en sus inicios - y en gran parte posteriormen- 
te- suelen estar más cercanos a la comunidad y pueden tomar mejor en cuenta las necesidades de las bases que representan. Pero, por otro lado, muchas veces carecen de incidencia sobre las políticas públicas y de vínculos con la comunidad internacional (Lederach, 2013: 12; Mouly, 2004). Mientras tanto, iniciativas más institucionalizadas, como las mesas cuando llegaron a ser parte de la institucionalidad de la paz, tienen mayor incidencia en las políticas públicas y pueden recibir el apoyo de la comunidad internacional. No obstante, esto puede llevarles a perder su vinculación con las bases. Como van Tongeren (2013) señala, la informalidad y la formalidad tienen fortalezas y debilidades. Las infraestructuras para la paz informales suelen enmarcarse en procesos más autóctonos y, por tanto, suelen contribuir a una paz más legítima (Richmond, 2012), pero muchas veces no tienen mayor impacto en el Estado y la sociedad. Las infraestructuras para la paz más institucionalizadas suelen tener vínculos con el Estado y otros actores clave y pueden tener una mayor incidencia en la construcción de la paz. Sin embargo, tienden a ser más dependientes del Estado y de los actores internacionales y menos a la escucha de las necesidades de la población local (Lederach, 2013).

Por consiguiente, el equilibrio es deseable para apoyar mejor la implementación de la paz. Esto significa, a nivel departamental, la creación de espacios con representación de los diferentes sectores sociales que conforman cada departamento, que estén lo suficientemente institucionalizados para tener incidencia en la formulación de políticas públicas, sin dejar de lado su vinculación con las bases, y lo suficientemente dinámicos para adaptarse a situaciones cambiantes. La metáfora de la telaraña introducida por John Paul Lederach (2008: 134-135) describe adecuadamente el tipo de infraestructura para la paz que se requiere: una con suficiente flexibilidad para adaptarse a su entorno. Para él "[1]a clave de la sostenibilidad no es la fortaleza masiva o una mayor fuerza - sea cual fuera la naturaleza de esa fuerza. Es la adaptabilidad.” Además, como lo plantea Oliver Richmond, las iniciativas locales de paz tienden a moverse de lo "escondido" a lo "informal" y lo "formal" (Richmond, 2012), como sucedió en las mesas de concertación en Guatemala. Las primeras mesas aparecieron a nivel departamental/regional como espacios informales en los que se encontraron varios sectores sociales para discutir la implementación de los acuerdos de paz, y solo tuvieron reconocimiento a posteriori. Este reconocimiento surgió del interés de la Misión de Naciones Unidas en Guatemala (MINUGUA), con presencia en todo el territorio, y su contraparte guatemalteca, la Comisión de Acompañamiento de los Acuerdos de Paz, en usar estos espacios para descentralizar la implementación de la paz y contribuir así a un proceso más incluyente que reflejara la diversidad del país. Por consiguiente, apoyaron los espacios existentes y promovieron la creación de mesas de concertación en los demás departamentos del país, a excepción del departamento de la capital. Además, vincularon las mesas a las instituciones creadas en virtud de los acuerdos de paz para apoyar su implementación. 
En contraposición al ejemplo de las mesas de concertación está el de los comités locales de paz establecidos en cada distrito de Nepal a instigación del gobierno central para descentralizar la implementación de la paz y abarcar la diversidad territorial. Estos comités fueron impulsados por el ministerio de Paz y Reconstrucción y contaron con el apoyo de la Misión de Naciones Unidas en Nepal (UNMIN). Sin embargo, esta dependencia del ministerio de Paz y Reconstrucción fue problemática porque los comités locales de paz se politizaron y sufrieron muchos cambios a raíz de la inestabilidad política caracterizada por frecuentes cambios en gobiernos y parálisis institucional en los años que siguieron la firma de los acuerdos de paz. Por consiguiente, los comités locales de paz nepaleses se volvieron presos de la política nacional al detrimento de su pertinencia a nivel local (Odendaal, 2010). Personas con mucha experticia y compromiso con la construcción de paz tuvieron que salir porque habían sido nombradas para ser parte de los comités locales de paz durante otros gobiernos. Esto significó que, salvo unas pocas excepciones, los comités locales de paz no estuvieron a la altura de la misión que se les había confiado. Fue una oportunidad desperdiciada de descentralizar el proceso de paz en Nepal. Como este ejemplo lo muestra, si una infraestructura para la paz es vista como impuesta sobre la sociedad en lugar de emanar de la misma, puede perder legitimidad y trascendencia.

Estas experiencias apuntan a la necesidad de tomar en cuenta las estructuras desarrolladas por varias iniciativas locales de construcción de paz que surgieron durante el conflicto armado en Colombia y evitar desplazar a formas organizativas anteriores, como ocurrió cuando la Comisión de Acompañamiento de los Acuerdos de Paz, por medio de la Secretaría de la Paz, impulsó el establecimiento de mesas de concertación en algunos departamentos. Por ejemplo, las iniciativas de territorio de paz que han emergido para hacer frente a la violencia perpetrada por los distintos grupos armados que operan en Colombia, muchas de las cuales han recibido apoyo de la Red Nacional de Iniciativas por la Paz y contra la Guerra (REDEPAZ), merecen ser consideradas a la hora de desarrollar una infraestructura para la paz. Varios territorios de paz han tenido logros innegables en materia de reducción de la violencia, como la comunidad de paz de San José de Apartadó (Masullo, 2015) o la asamblea constituyente de Mogotes (García Durán \& Sarmiento, 2015), y han demostrado su sostenibilidad a través de los años. Otras iniciativas, como la Asociación de Trabajadores Campesinos del Carare (ATCC) en La India (Hernández, 2013; Kaplan, 2013), o los programas de desarrollo y paz, como el Programa de Desarrollo y Paz del Magdalena Medio con sus distintos componentes (García \& Llinás, 2012), también pueden aportar mucho a la paz territorial. Estas experiencias no solamente deberían encontrar su lugar en una infraestructura para la paz, sino servir de modelos para otras localidades que pueden beneficiarse del intercambio de experiencias y lecciones aprendidas. La experiencia exitosa de desminado huma- 
nitario en Samaniego, por ejemplo, se benefició del intercambio con la experiencia exitosa de Mogotes (García Durán \& Sarmiento, 2015; Idler, Garrido, \& Mouly, 2015; Mouly, Garrido, \& Idler, 2016). Estas iniciativas a nivel local deberían ser aprovechadas y apoyadas desde el nivel departamental y articularse con ese nivel.

Finalmente, se debe recordar que, más allá del equilibrio entre institucionalización e informalidad, lo más importante no es la sostenibilidad de una infraestructura para la paz, cuyo rol puede ser transitorio; sino los procesos que esta infraestructura desencadena o consolida y cómo contribuyen a la construcción de un Estado y una sociedad más incluyentes y tolerantes, con mejores capacidades para transformar los conflictos de manera pacífica (Mouly, 2013). En efecto, este debe ser el objetivo de la construcción de paz a largo plazo y pasa por cambios a nivel estructural y cultural que pueden llevar muchos años.

\section{Conclusiones}

Este artículo recogió lecciones aprendidas de la experiencia de las mesas de concertación en Guatemala que pueden servir para la paz territorial en Colombia. Esta discusión es necesaria en el contexto actual para garantizar el éxito del proceso de implementación de la paz. En efecto, la implementación de la paz no puede ser impuesta. Requiere la participación y apropiación de los diversos sectores de la población colombiana en las distintas localidades del país.

En primer lugar, vimos cómo las mesas de concertación en Guatemala llegaron a conformar una infraestructura para la paz con tres niveles: el nacional, el departamental y el municipal. El nivel departamental jugó un papel estratégico para articular las necesidades de la población local en distintas partes del territorio con los procesos de toma de decisión a nivel nacional. Este modelo puede ser interesante para la paz territorial en Colombia, de manera que se genere mayor apropiación en el proceso de implementación de la paz, y se aborden las diversas necesidades de la población en los distintos rincones del país. Será necesario tratar de evitar duplicidades y sacar provecho de los mecanismos e iniciativas existentes cuando sea posible, tomando en consideración las realidades de cada localidad.

En segundo lugar, discutimos acerca de la participación de los grupos tradicionalmente marginados en las mesas de concertación y del rol de intermediación de las mesas. Esta discusión también aportó insumos para la paz territorial en Colombia. Se argumentó que la participación de grupos históricamente marginados, como indígenas, afrocolombianos, campesinos o mujeres, es esencial en el proceso de implementación de la paz. También se enfatizó la necesidad de tomar en cuenta las iniciativas de paz que estos grupos han impulsado a nivel local. Por otro lado, se discutió el valioso rol de 
intermediación que pueden tener espacios multisectoriales de concertación en Colombia. Pueden acercar a diversos grupos sociales y facilitar la transformación pacífica de conflictos locales, como ocurrió en Guatemala, especialmente cuando no existe la capacidad o legitimidad para canalizar estos conflictos por medio de instituciones estatales.

En tercer lugar, argumentamos que se debería mantener un balance entre informalidad e institucionalización en el desarrollo de una infraestructura para la paz de alcance nacional en Colombia. Esto permitirá mantener una vinculación con las bases sociales y al mismo tiempo tener más incidencia en las decisiones tomadas a nivel central, en particular en las políticas públicas. No es imprescindible que espacios como las mesas sean sostenibles para ser exitosos: es más importante que contribuyan a fortalecer las capacidades del Estado y la sociedad para hacerlos más incluyentes, más tolerantes y más capaces de transformar los conflictos de forma pacífica.

Este artículo se ha enfocado en las lecciones que las mesas de concertación aportan en términos organizacionales para la paz territorial en Colombia. Sería útil reflexionar más acerca de las actividades que deberían ser impulsadas en el marco de la paz territorial. El caso de las mesas de concertación guatemaltecas ofrece algunos insumos útiles en cuanto a actividades de difusión de los acuerdos de paz (ej. foros municipales y departamentales, programas radiales) y de incidencia en leyes y políticas públicas (ej. encuentros con diputados, propuestas canalizadas a través de la Comisión de Acompañamiento de los Acuerdos de Paz o de los consejos de desarrollo), pero sería bueno profundizar esta discusión a la luz de las experiencias exitosas de construcción de paz que se han venido desarrollando a distintos niveles en Colombia. Finalmente, valdría la pena reflexionar más sobre el rol del Estado. Los acuerdos de paz seguramente incluirán pautas para la creación de mecanismos institucionales, leyes y políticas que el Estado debería adoptar para incorporar los aportes de los gobiernos y las sociedades civiles locales en la implementación de la paz. Y estas probablemente habrán sido objeto de una amplia discusión a la luz de experiencias pasadas. Sin embargo, como se mencionó antes, los acuerdos de paz no pueden abarcarlo todo. Siempre existen vacíos que se deberán llenar y detalles que se deberán especificar en el momento de la implementación. Por lo tanto, la discusión debe quedar abierta y seguir nutriéndose de los aportes de todos y todas. 


\section{Referencias}

ACUERDO GENERAL PARA LA TERMINACIÓN DEL CONFLICTO Y LA CONSTRUCCIÓN DE UNA PAZ ESTABLE Y DURADERA (2012). Firmado por el gobierno colombiano y las FARC el 26 de agosto de 2012 en La Habana. Recuperado de: https://www.mesadeconversaciones.com.co/ sites/default/files/AcuerdoGeneralTerminacionConflicto.pdf

ACUERDO SOBRE ASPECTOS SOCIOECONÓMICOS Y SITUACIÓN AGRARIA (1996). Firmado por el gobierno guatemalteco y la URNG el 6 de mayo de 1996 en México D.F. Recuperado de: http:// old.congreso.gob.gt/Docs/PAZ/ACUERDO\%2OSOBRE\%2OASPECTOS\%2OSOCIOECON\%C3\%93MICOS\%2OY\%2OSITUACI\%C3\%93N\%2OAGRARIA.pdf

AGUILAR, I. (2002). "La buena voluntad al servicio de la paz: La experiencia de la Mesa de Negociaciones para Conflictos de Tierra en Alta Verapaz, Guatemala". En Y. A. Soto (Ed.), Nuevos caminos para la resolución de conflictos: experiencias latinoamericanas (pp.107-140). Guatemala: Novedades.

COMISIÓN DE ESCLARECIMIENTO HISTÓRICO. (1999). "Guatemala: Memoria del Silencio. Conclusiones y recomendaciones del Informe de la Comisión para el Esclarecimiento Histórico". Guatemala: Comisión para el Esclarecimiento Histórico.

DECRETO 11-2002, LEY DE CONSEJOS DE DESARROLLO URBANO Y RURAL (2002).

CHOPRA, T. (2009). "When Peacebuilding Contradicts Statebuilding: Notes from the Arid Lands of Kenya”. International Peacekeeping, 16(4), 531-545. doi: 10.1080/13533310903184721

DANE. (s/f). “La visibilización estadística de los grupos étnicos colombianos”. Bogotá: Departamento Administrativo Nacional de Estadística.

DONAIS, T. (2009). "Empowerment or Imposition? Dilemmas of Local Ownership in Post-Conflict Peacebuilding Processes". Peace \& Change, 4(1). doi: 10.1111/j.1468-0130.2009.00531.x

GARCÍA, A., \& LLINÁS, G. (2012). "Los Programas de Desarrollo y Paz (PDP) de Colombia: una apuesta innovadora por el desarrollo y la paz en medio del conflicto armado”. In A. Rettberg (Ed.), Construcción de paz en Colombia (pp. 273-304). Bogotá: Universidad de los Andes.

GARCÍA DURÁN, M., \& SARMIENTO, F. (2015). “Demining in Micoahumado: from civil resistance to local negotiation with the ELN". In S. Haspeslagh \& Z. Yousuf (Eds.), Local engagement with armed groups: In the midst of violence. Accord Insight 2 (pp. 21-26). Londres: Conciliation Resources.

GHEBREMSKEL, A., \& SMITH, R. (2013). “Comments on Paul van Tongeren's 'Potential cornerstone of infrastructure for peace? How local peace committees can make a difference". Peacebuilding, $1(1), 65-68$.

HERNÁNDEZ, E. (2012). Intervenir antes de que anochezca: Mediaciones, intermediacionesy diplomacias no violentas de base social en el conflicto armado. Bogotá: La Bastilla. 
HERNÁNDEZ, E. (2013). "Mediaciones en el conflicto armado colombiano: hallazgos desde la investigación para la paz". CONfines, 9(18), 33-57.

HOPP-NISHANKA, U. (2012). "Giving Peace an Address? Reflections on the Potential and Challenges of Creating Peace Infrastructures”. In B. Unger, S. Lundström, K. Planta \& B. Austin (Eds.), Peace Infrastructures - Assessing Concept and Practice. Berghof Handbook Dialogue No.1o. Berlin: Berghof Foundation.

IDLER, A., GARRIDO, M. B., \& MOULY, C. (2015). "Peace Territories In Colombia: Comparing Civil Resistance In Two War-Torn Communities". Journal of Peacebuilding \& Development, 10(3), 1-15. doi: 10.1080/15423166.2015.1082437

IDLER, A., MOULY, C., \& MIRANDA, L. (2015). "Power Unpacked: Domination, Empowerment and Participation in Local Guatemalan Peace Forums". Peace, Conflict \& Development (21), 1-40.

JARAMILLO, S. (2014). "La paz territorial”. Presentación en la Universidad de Harvard, Cambridge, MA, Estados Unidos, 13 de marzo.

KAPLAN, O. (2013). "Protecting civilians in civil war: The institution of the ATCC in Colombia". Journal of Peace Research, 50(3), 351-367. doi: 10.1177/0022343313477884

LEDERACH, J. P. (1997). Building peace: sustainable reconciliation in divided societies. Washington, D.C.: United States Institute of Peace Press.

LEDERACH, J. P. (1999). “Justpeace". En European Centre for Conflict Prevention, in cooperation with IFOR \& the Coexistence Initiative of the State of the World Forum (Eds.), People Building Peace: 35 Inspiring Stories from Around the World (pp. 27-36).

LEDERACH, J. P. (2008). La imaginación moral: el arte y alma de construir la paz. Bogotá: editorial Norma.

LEDERACH, J. P. (2013). “The Origins and Evolution of Infrastructures for Peace: A Personal Reflection”. Journal of Peacebuilding \& Development, 7(3), 8-13. doi: 10.1080/15423166.2013.767604

MASULLO, J. (2015). The Power of Staying Put: Nonviolent Resistance against Armed Groups in Colombia. Washington D.C.: International Center on Nonviolent Conflict.

MDC DE JUTIAPA, MDC DE CHIMALTENANGO, MDC DE ALTA VERAPAZ, \& MDC DE IZABAL. (2003). "Estatutos de la Asamblea Nacional de Mesas de Concertación, ANAMEC; coordinadora nacional de mesas de concertación, CONAMEC y mesas departamentales de concertación, MDC y Seguimiento de los Acuerdos de Paz". Izabal.

MESAS DE SACATEPÉQUEZ, QUETZALTENANGO, SUCHITEPÉQUEZ, \& EL PROGRESO. (2001). "Encuentro Nacional de Mesas Departamentales de Concertación y Seguimiento de los Acuerdos de Paz, La Antigua Guatemala".

MOULY, C. (2004). The Role of Peace Constituencies in Building Peace in Nicaragua and Guatemala. Tesis de doctorado (PhD), Universidad de Cambridge, Cambridge. 
MOULY, C. (2013). “The Nicaraguan Peace Commissions: A Sustainable Bottom-Up Peace Infrastructure”. International Peacekeeping, 20(1). doi: 10.1080/13533312.2012.761833

MOULY, C., GARRIDO, M. B., \& IDLER, A. (2016). “How Peace Takes Shape Locally: The Experience of Civil Resistance of Samaniego in Colombia”. Peace \& Change, 41(2), 129-166. doi:10.1111/pech.12184

ODENDAAL, A. (2010). "An Architecture for Building Peace at the Local Level: A Comparative Study of Local Peace Committees" Discussion paper. Nueva York: PNUD.

PNUD. (1998). Guatemala: Los Contrastes del Desarrollo Humano. Guatemala: PNUD.

RICHMOND, O. P. (2012). “Missing Links: Peace Infrastructures and Peace Formation”. In B. Unger, S. Lundström, K. Planta \& B. Austin (Eds.), Peace Infrastructures - Assessing Concept and Practice. Berghof Handbook Dialogue No.1o. Berlin: Berghof Foundation.

ROJAS, C. (2009). "Women and peacebuilding in Colombia: resistance to war, creativity for peace". In V. M. Bouvier (Ed.), Colombia: building peace in a time of war (pp. 207-224). Washington: United States Institute Peace Press.

ROMERO, M. (2015, 7 de febrero). Desafíos de la paz territorial. UNPeriódico, recuperado de: http:// www.unperiodico.unal.edu.co/dper/article/desafios-de-la-paz-territorial.html

UGARRIZA, J.E. \& NUSSIO, E. (2016) “The Effect of Perspective-Giving on Postconflict Reconciliation. An Experimental Approach”, Political Psychology,. doi: 10.1111/pops.12324.

VAN TONGEREN, P. (2013). "Potential Cornerstone of Infrastructures for Peace? How Local Peace Committees Can Make a Difference”. Peacebuilding, 1(1),39-6o. doi:10.1080/21647259.2013.756264 